zur Verwendung brauchbar wurde. Die edlen Leute waren so frei von jedem Eigenuutz, dass ich ihrer stets mit wohlverdienter Dankbarkeit gedenken werde.

(Fortsetzung folgt.)

\title{
Berichtigungen und Nachträge zur Flora von Mähren.
}

\section{Von Joh. Bubela.}

Nachdem die Ausgabe der "Flora von Mähren and ôsterr. Schlesien" von Ad. Oborny bereits zur Vollendung gelangt ist, fühle ich mich verpflichtet, auf einf́ge Irrthümer bezüglich jener Standorte hinzuweisen, die der Verfasser aus meinen brieflichen Mittheilungen und Publicationen schöpfte. Einigo dieser Irrthümer beruhen auf unrichtiger Bestimmung der Pflanzen meinerseits, andere wiederum auf verschiedenartigen Versehen von Seite des Autors.

Hiezu füge ich auch alle jene Funde bei, die ich in den letzten Jahren in diversen Gegenden machte, so auch alle jene Standorte, die ich bei Durchsicht meines Herbars und einiger älteren Publicationen ermittelte.

Wsetin, 4. September 1887.

Ad Nr. 3. Polypodium Robertianum A. Br. Wsetin: selten an Mauern der herrsch. Brücke.

14. Asplenium septentrionale Hffm. Wsetin: „Č́rtova skála“" (Tèufelsfelsen) bei Lidečko.

15. - germánicum Weiss. Oels: Skřipského stráň (Člupèk).

25. Aspidiwm filix mas Sw. a) genuinum Mde. Um Wsetin; b) erenatum Mde. Waldstrecke Bystrìcky; c) incisum Mde. Waldstrecke Huboký boi Wsetin.

32. Botrychium lunaria Sw. Sehr selten auf der "Čertova skála" bei Lidečko.

37. Equisetum maximum Lmk. Bei Weisskirchen (Sapetza).

38. - silvaticum L. Grosser Kessel im M. Gesenke.

42. - palistre L. e) polystachyum Vill. Bei Wsetin.

43. - ramosissimum Dsf. b) gracile A. Br. Wsetin: na Trávníkách und an der Strasse nach Lásky.

48. Lycopodium clavatum L. Kř́ǐ̌ový-Berg bei Wsotia.

49. - complanatum L. Fichtencultur oberhalb Waldenburg bei Freiwaldau.

64. Lemna gibba L. Im Gevatterloch bei Weisskirchen fand ich bei genaner Untersuchung nur L. minor!

91. Panicum glabrum Gand. Letovice (Člupek).

96. Setaria verticillata Beauv. Weinberge bei Bisenz. 
115. Alopecurus geniculatus L. var. nigricans (mit schwarz überlaufenen Aehren) bei Wsetin.

120. Phleum pratense L. $\beta$ ) nodosum L. Wsetin: Bobrky. Gräfenberg: Nesselkoppe.

134. Avena pubescens Hds. (non L.!) Wsetin: Feldraine oberbalb Lásky.

137. Aira caryophyllea L. Nach Vogl um Solanec bei Rožnau.

139. - Alexuosa L. Wsetin: sehr selten auf Wiesen "v Rybníkn“.

140. Trisetum flavescens P. B. Rottalowitz (Sloboda).

144. Danthonio decumbens DC. Moravské vrchy bei Oels (Člupek); bei Wsetin häufig.

149. Cynosures eristatus L. Fehlt bei Bisenz!

157. Poa palustris L. Wsetin: Bečvaufer.

164. Molinia coerulea Mnch. Bei Oels (Člupek), wodurch die Vermuthung Ob.: ,Scheint dem böhmisch-mährischen Scheidegebirge zu fehlen" widerlegt wird.

167. Glyceria fluitans $\mathrm{R}$. Br. Wsetin.

171. Festuca pseudo myurus Soy. W. Kiesbänke der Ostravica bei Mistek (Gogola). Neu für Mähren!

172. - ovina L. e) sulcata Hck. $\gamma$ ) valesiaca Koch. Wsetin: Wiesen "v Rybníku".

182. Bromus commutatus Schrad. Wsetin.

185. - arvensis L. Wsetin.

190. - asper Murr. Wsetin: Bečevná-Berg.

192. - erectus Hds. Wsetin: sehr selten auf Feldrainen oberhalb Lásky.

193. - inermis Leyss. Rottalovic (Sloboda).

196. Triticum caninum L. Wsetin: Weidengobüsch an der Bečra.

198. Lotium multiflorum Lmk. an der Bečva bei Wsetin früher, neuerer Zeit verschwunden.

200. - temulentum L. $\beta$ ) leptochaeton A. Br. Wsetin: häufig im Getreide.

211. Carex paradoxa Willd., "Foustkova louka" bei Oels (Člupek). 218. - elongata L. Wsetin: v Rybníku.

227. - Goodenoughii Gay. $\gamma$ ) juncella Fr. Wsetin: Sümpfe zwischen Rybník und Žmboška, f. decipiens (vulgaris $>$ panicea?) Fiek in litt. Daselbst.

229. - stricta Good. Rottalovic (Sloboda).

230. - caespitosa L. Was ich unter diesem Namen von D. Sloboda erhielt, war C. Goodenoughii.

234. - pilulifera L. "Cortovy skály" bei Lidečko.

242. - facea Schrb. Rottalovic (Sloboda), Wsetin.

255. - Oederi Ehr. Wsetin.

261. - rostrata With. Wsetin.

264. Carex hirta L. $\beta$ ) hirtaeformis Prs. Wsetin, mit der vorigen unterhalb Lásky "na panském". 
275. Scirpus setaceus L. Wsetin: Čup-Berg.

280. Heleocharis ovata R. Br. Der Wsetiner Standort ist zu streichen. 284. Eriophorum latifolium Hppe. Rottalovic (Sloboda).

287. Cyperus favescens L. Wsetin-Cup.

323. Ornithogalum tenuifolium Guss. Bisenz: im Walde Háj. (det. Uechtritz).

325. Scilla bifolia L. Jarcowa bei Wall.-Meseritsch.

326. Allium ursinum L. Bei Zauchtl (Szepészy).

337. Muscari comosum L. Bei Freistadtl häufig.

339. - racemosum L. kommt bei Bisenz gar nicht vor. Der Standort in Ob. Fl.: auf Feldern und Eisenbahndämmen um Bisenz ist irrthümlich hierher gesetzt worden, gehört jedoch zu $M$. comosum. (Vide mein Verzeichniss der um Bisenz wildwachsenden Pflanzen in Verh. der zool.-bot. Ges. Wien 1882.)

341. Asparagus officinalis L. Wsetin: auf einem Felde "u Lapačù".

344. Convallaria majalis L. Felsen des Gr.-Kessel im Gesenke.

345. Polygonatum officinale All. Bei Rottalovic (Sloboda). Der Standort "spärlich auch im Vesniker Thale und in Ratibor bei Wsetin" ist ebenfalls verschrieben und gehört zu Conv. majalis. Pol. officinale kommt bei Wsetin gar nicht vor.

346. - muitiflorum All. Statt: Hojná bei Wsetin soll es heissen: Häufig bei W.

349. Colchicum autumnale L. f. vernale Hffm. var. speciosissimum $\mathrm{m}$. (in Oesterr. bot. Zeitschr. 1884, pag. 425), auch bei Prossnitz (Spitzner), Freiberg (Remeš).

371. Orchis incarnata L. Der Standort: auf einer Wiese zwischen Bobrky und Vesník bei Wsetin gehört za $O$. maculata $L$. var. candidissima Krock (vide Oesterr. bot. Zeitschr. 1886, pag. 364). O. incarnata vereinzelt auf Wiesen "na Ohradë".

380. Epipogon aphyllus Sw. Gesenke: am Fusswege von Lindewiese aus gegen Hockschar zu im Hochwalde.

384. Epipactis rubiginosa Gaud. Der Wsetiner Standort gehört zu Ep. latifolia c) violacea D. D.

389. Goodyera repens R. Br. Gesenke: Im Walde beim Moosebruch nächst Reiwiesen.

391. Corallorrhiza innata R. Br. Oels (Člupek).

396. Iris germanica L. Schlosser's Standort: "hie und da in den Karpathengegenden " bezieht sich gewiss nur auf cultivirte Pflanzen der Bauerngärten, sonst kommt diese Art in den Karpathen gar nicht vor.

408. Galanthus nivalis L. Jarcowa bei W.-Meseritsch.

416. Eaphorbia falcata L. Jasenná bei Wisovic.

420. - stricta L. Häufig um Wsetin und Wisovic.

434. Mercurialis ovata Stb. und H. Der Wsetiner Standort beruht auf falscher Bestimmung, ist demnach zu streichen.

439. Alnus viridis DC. Boi Oels (Člupek). 
455. Salix daphnoides Vill. Rottalovic (Sloboda).

471. Ulmus campestris L. (U. montana Sm.) Wsetin: bei Lásky und sonst vereinzelt.

501. Albersia blitum Kunth. Bei Wsetin, Wisoric und Bisenz.

529. Stellera passerina L. Jasenná bei Wisovic.

538. Loranthus europaeus Jcq. Im Walde Háj bei Bisenz.

541. Plantago major L. var. crispa m. (Niedrig; Blätter dicklich, in der unteren Hälfte am Rande grob gezähnelt und wellig.) Am Gipfel des Radhost.

543. - lanceolata L. 8) sphaerostachya M. und K. Bergwiesen am Radhost.

548. Mentha caudicans Cr. $\beta$ ) mollissima Bkh. Wsatin.

550. - palustris Mnch. $\gamma$ ) ballotaefolia Op. (det. Braun). Bečvaufer bei Wsetin. $\delta$ ) subspicata Whe. Um Wsetin, Wisovic.

- Hyssopus officinalis L. An Mauern des evangelischen Friedhofes in Wisovic häufig.

573. Nepeta cataria L. An Rainen in U.-Jasenka bei Wsetin.

576. Galeopsis ladanum L. $\beta$ ) angustifolia Ehr. Häufig um Wsetin und Wisovic.

582. Stachys alpina L. Teplicer Wälder bei Weisskirchen.

586. - annua L. Rottalovic (Sloboda).

587. - recta L. Jankovice bei Rottalovic (Sloboda).

594. Leonurus cardiaca L. Fehlt um Wsetin, Rożnau und Wall.Meseritsch.

599. Marrubium vulgare L. Lutonina bei Wisovic.

606. Ajuga genevensis L. Um Wsetin auch roth und weiss blühend. Der Zivotsky'sche Standort der A. pyramidalis L. "auf der Vlkoška bei Wsetin" beruht gewiss auf Verwechslung mit $A$. genevensis.

608. Teucrium botrys L. Bei Rottalovic kommt diese Pflanze gewiss nicht vor, die Angabe Slob oda's bezieht sich vermuthlich auf die folgende Art, die ich von dort anch besitze.

610. - chamaedrys L. Rottalovic (Slob.), höchst selten auf den "Travníky" bei Wsetin.

619. Orobanche major L. in der Form Kochii F. Sz. Nikolčic bei Auspitz (Sebesta) vide Oesterr. bot. Zeitschr. 1882, pag. 117.

626. Melampyrum cristatum L. soll es heissen: Bergwiesen um Wsetin, nicht „Horní luka".

629. - pratense L. Fehlt um Wsetin.

639. Rhinanthus serotinus Schönh. Um Wsetin, Bobrky und oberhalb der Glashütte auf Bergwiesen.

661. Veronica triphylla L. Fehlt bei Wsetin.

667. Digitalis ambigua Murr. Rottalovic (Slob.), Francova Lhota (Merlícek), Wsetin: Johanovský Láz.

680. Scrophularia Scopolii Hppe. Selten um Lásky boi Wsetin.

694. Physalis alleekengi L. Zopy bei Rottalovic (Sloboda).

713. Gentiana verna L. Gesenke: Am. Wego vom Altvater zur Schäferei. 
717. Gentiana obtusifolia W. Häufig auf Wiesen hinter Hośtialkau bei Wsetin.

721. Erythraea ramosissima Prs. Wsetin: Sümpfe am Čp-Berge.

724. Cynoglossum officinale L. Wsetin: sehr selten bei Ustí.

728. Myosotis sparsiflora Mik. Wsetin: an Wegen im Schlossparke. 729. - caespitosa F. Sz. Wsetin: V̌́eliniska.

734. - versicolor Sm. Wsetin: Bobrky.

748. Anchusa officinalis L. Fehlt um Wsetin. Um Weisskirchen.

788. Chimophila umbellata Nutt. Sapetza's "Teufelsmühle". ist zweifelsohne jone unweit von Nentitschein, nicht aber die Teufelsmühle "bei Czeladna".

803. Campanula glomerata L. Hăfig bei Wsetin.

816. Arnoseris minima Lk. Nach Slob oda's „Rostlinnictvi“ pag. 451 um Nentitschein.

826. Crepis paludosa Mnch. Selten im Vesníker Thal bei Wsetin. 830. Hieracium flagellare W. Wsetin: Bobrky und Rybnik, häufig. $829 \times 836$. - pilosella praealtum Nlr. b) Bauhini $\times$ pilos. (U日eht.) Am Wsetiner Friedhofe.

836. - praealtum Koch var. glaberrimum m. (Blätter, Stengel und Hüllen ganz kahl, diese klein, ganze Pflanze steif.) Wsetin: am Čp-Berge.

838. - cymosum L. f. glomeratum Fröl. Wsetin: Vlkoška.

856. - murorum L. $\beta$ ) microcephalum Uecht. Wsetin. Annäherungsformen von $\boldsymbol{H}$. cinerascens Jord. im Walde Poschlá bei Wsetin.

857. vulgatum Fr. d) argutidens Fr. in einer Annäherungsform „za Díly" bei Wsotin.

861. - tridentatum Fr. $\gamma$ ) angustifolium Uecht. Wsetin: Vesnik.

875. Sonchus arvensis L. b) uliginosus M. B. Um Wsetin.

878. Prenanthes purpurea L. Um Teplic bei Weisskirehen.

931. Achillea ptarmica Trnf. Am Wege zwischen Jeleňová und dem Nivka-Berge bei Wsetin.

940. Arthemis tinotoria L. Wsetin: sehr spärlich "nade Mžíky“.

954. Filago canescens Jord. Um Wisovic.

959. Gnaphalium norvegicum Gunn. Gesenke: Nesselkoppe bei Gräfenberg.

964. Doronicum austriacum Jeq. Radhošt (Sloboda).

981. Senecio crispatus DC. $\beta$ ) rivularis Rchb. f. sudetica Koch. (?) (Nach einem unvollständigen Exemplare bestimmt.) Francova Lhota.

(Schiuss folgt.)

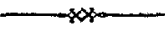

\section{Iiteraturberichte.}

Prof. Dr. Schenk: Fossile Pflanzen aus der Alburskette. Gesammelt von E. Tietze, Chefgeologen der k. k. geolog. Reichsanstalt. Mit 9 Tafeln. (Heft Nr. 6 der Bibliotheca botanica.) Abhandlungen aus dem Ge- 